\title{
Imaging of Stellar Disks and Mass Loss Envelopes in Evolved Stars
}

\author{
P.G. Tuthill, J.D. Monnier \& W.C. Danchi \\ Space Science Laboratory, University of California, Berkeley, CA 94720, \\ $U S A$
}

\begin{abstract}
Interferometric imaging of evolved stars has been performed in the near-IR at the Keck Observatory revealing diffraction-limited structure at unprecedented spatial resolution. By studying the hottest regions of the circumstellar dust shells of a number of bright prototypical objects, we have revealed complex and anisotropic structures in the inner regions where dust nucleation occurs. We find that dramatic dust plumes and clumps dominate the morphology of the circumstellar environment at a scale of a few stellar radii for extreme carbon stars such as IRC +10216 and CIT 6. Furthermore our observations have yielded near-IR diameters and images of the stellar photospheres of nearby red giant and supergiant stars. With the additional benefit of multi-epoch observations, we have begun to investigate the time-evolution of our enshrouded objects as dust is created and accelerated away from the nucleation zone. Armed with such a powerful new set of observational constraints, we are able to address contemporary problems such as the origin of structure in $\mathrm{PNe}$, the physics of mass loss, and the dynamical properties of Mira pulsation.
\end{abstract}

\section{Introduction}

Interferometry in the infrared is providing a new observational window on the environments of late type stars from the stellar photospheres out to the extended circumstellar dust shells. The resolution attainable with the latest generation of $10 \mathrm{~m}$ class telescopes is well suited to the study of the nearest and brightest dust-enshrouded stars. This paper describes a program running at the Keck observatory over the last few years obtaining images of bright evolved stars at the diffraction limit of the Keck aperture. For these types of objects, studies in the near-IR are particularly profitable. This is due to the low dust opacity, the low contamination from molecular lines, and the high luminosity of the sources. Coupled with this, the hottest dust in the inner regions of the dust shells becomes bright enough so that the blackbody emission can rival or even outshine the radiation coming from the parent star, enabling us to make images of the flux coming from this circumstellar material.

Using multi-wavelength images spanning the infrared JHK and L bands, we have begun to address some of the most pressing questions related to the latest stages of stellar evolution. Our observations of a handful of bright evolved LPV's, supergiants and enshrouded stars have allowed us to explore the processes underlying mass loss, a key feature of such evolved systems. Although 
theoretical models usually assume spherical symmetry, there has been a growing body of evidence (e.g. from molecular and polarization data, spectrophotometry, and observations of planetary nebulae) that mass loss is often anisotropic. Our high-resolution images have provided some of the newest insights into this phenomenon.

\section{Aperture masking interferometry}

Our observations have utilized the technique of aperture masking, in which a mask is placed over the telescope pupil so as to only pass light from selected regions, thus transforming the telescope into an array of small subapertures. When the light from each of these separate subapertures is combined, interference fringes are formed which encode information on the spatial structure of the source object. The masking of the Keck 1 primary was achieved by mounting custom-made plates in front of the $\mathrm{f} / 25$ secondary mirror. An example showing the hole-pattern of our 15-hole mask, as projected onto the Keck primary mirror, is given in Fig. 1. There are many constraints in arriving at such pupil geometries, with the primary concern being to sample the Fourier plane evenly and densely out to the cut-off of the telescope, in this instance with a non-redundant array - a sampling in which no vector baseline occurs more than once.
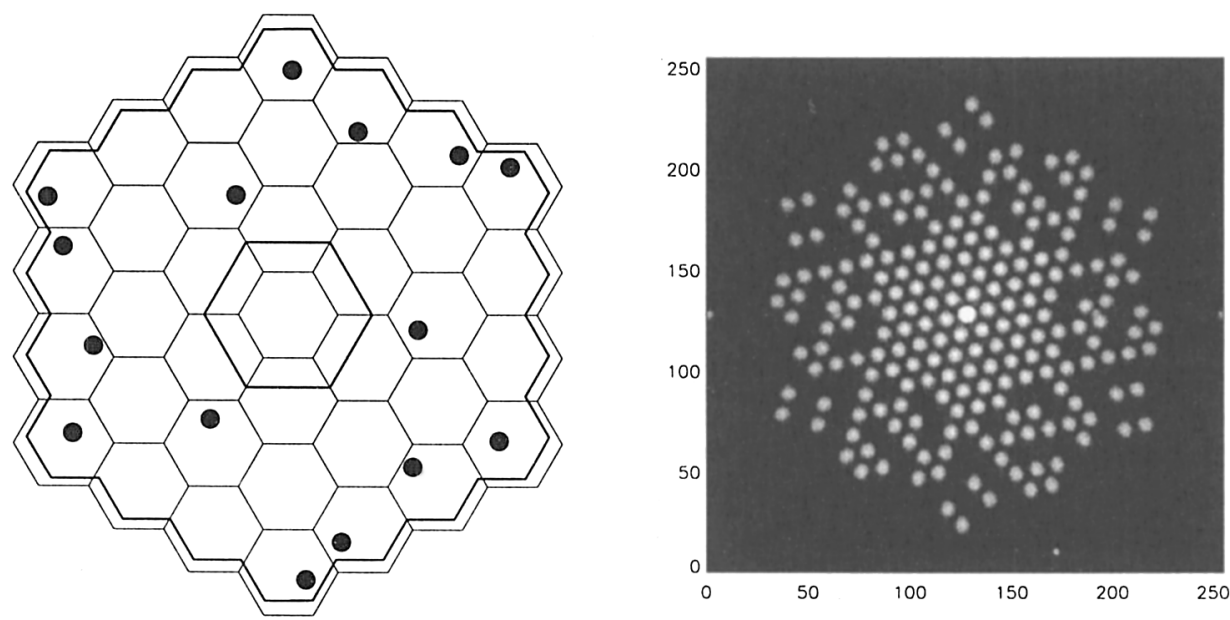

Figure 1. A 15-hole non-redundant array as it appears projected onto the Keck primary mirror (left) and the corresponding power spectrum obtained by Fourier analysis of the resultant fringe pattern (right).

After passing through the mask, starlight was brought to focus in the Near Infra-Red Camera (NIRC), a cryogenically cooled $256 \times 256$ pixel InSb array 
(Matthews \& Soifer 1994). Using the NIRC magnifying optics (Matthews et al. 1996) gave a pixel scale of 20.57 milli-arcsec/pixel - sufficient to Nyquist sample fringes on the longest baselines in K-band. With the aperture mask in place, the image formed at the camera focus is an Airy disk crossed by multiple sets of fringes corresponding to each pair of holes in the mask. In order to preserve as much high-resolution information as possible in this rapidly-evolving pattern, the array was clocked out in a 'speckle' readout mode as rapidly as possible resulting in data cubes of 100 consecutive frames each with $140 \mathrm{msec}$ exposure time.

Data reduction proceeded by extracting the visibilities and phases from each exposure which are related to the brightness distribution of the science target by a Fourier transform (e.g. Born \& Wolf 1964). Fourier amplitudes may be conveniently extracted from the power spectrum, an example of which is given in Fig. 1. Although the Fourier phases were corrupted by atmospheric fluctuations, an observable known as the closure phase - the sum of baseline phases around closed triangles - has been developed in radio astronomy to overcome this problem. These techniques are now well established at optical wavelengths (Haniff et al. 1987; Roddier 1988).

Raw visibilities were calibrated for the mean telescope-atmosphere transfer function by utilizing nearly contemporaneous observations of point-source stars. After such calibration, Fourier data were inverted to yield images with the help of reconstruction algorithms such as the "Maximum Entropy Method" (Gull \& Skilling 1984; Sivia 1987) or CLEAN algorithm (Högbom 1974) - both of which have been used extensively in radio astronomy.

Both theoretical and experimental studies have highlighted the gains in signal-to-noise ratio (SNR) made available by using sparse rather than filled apertures in interferometric observations of bright sources (Roddier 1988; Haniff $\&$ Buscher 1992). Our own results have verified these predictions, and enabled us to reconstruct complex images at diffraction-limited angular resolution in the near-infrared.

\section{Results}

This program has extensive data on a wide variety of Supergiants, Miras and enshrouded evolved stars. The supergiant VY CMa has been found to exhibit a one-sided extension at high resolution in the near-IR which appears to be linked to a curved plume outflow in the extended circumstellar environment. Further details on this fascinating system can be found in Monnier et al. (1999). Rather than trying to summarize observations of dozens of stars, and to give a flavor of this work within the constraints of this report, we focus here on two famous bright objects: $o$ Ceti and IRC +10216 .

Our diffraction-limited observations with the Keck telescope have enabled us to measure photospheric stellar diameters in a number of near-IR wavebands, as is illustrated in Fig. 2 for $o$ Ceti, the prototype Mira variable. Such highquality visibility data allows us to address a number of important topics in the study of evolved stars.

The angular diameter is amongst the most fundamental stellar observables; from it may be computed the effective temperature and the luminosity. Fur- 
o Ceti visibility curves (Dec 97)

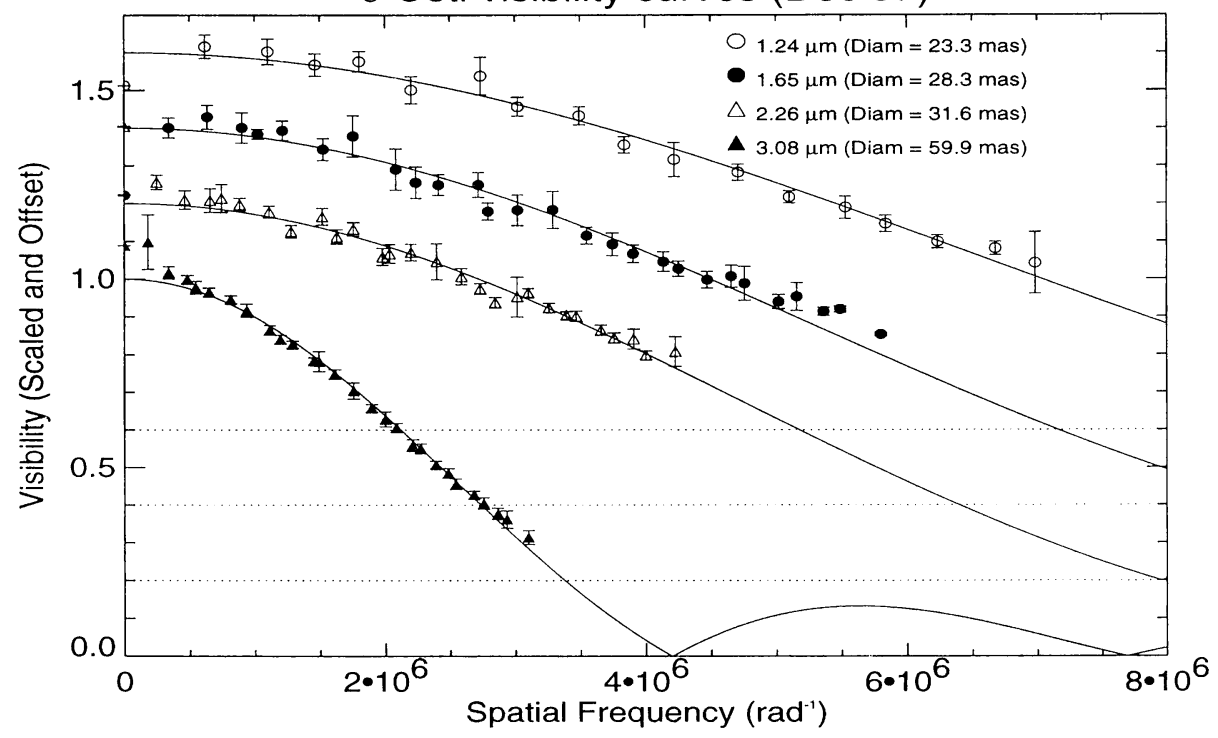

Figure 2. Azimuthally averaged visibility curves produced for $o$ Ceti from multi-wavelength interferometer data (vis offsets have been added to avoid confusion on the plot). Diameters given correspond to the best-fit uniform-disk models. The larger apparent diameter recorded at $3.08 \mu \mathrm{m}$ may be due to molecular blanketing around the star.

thermore, in cases where stars are known to be pulsating, the diameter can be used for dynamical studies such as those of Haniff et al. (1995) to determine the pulsation mode - a question which remains open despite extensive debate. Additional data forming a time-series record of stellar diameters through the pulsation cycle should be able to directly observe changes in photospheric radius, greatly clarifying observational aspects of stellar pulsation in LPV's.

Our quasi-monochromatic diameters at a range of different wavelengths probe the atmospheric structure, helping to discriminate emission from the photosphere, molecular atmosphere and circumstellar dust, thereby providing valuable experimental testing of numerical model atmospheres. We report here a particularly marked enlargement in the apparent size of $o$ Ceti in the $3.08 \mu \mathrm{m}$ filter, an effect we believe is due to absorption of the stellar flux by some molecular species, probably water. Clearly, such data is best interpreted in the context of a numerical simulation involving radiative transfer through a stellar atmosphere with appropriate abundances of molecular and atomic species.

As a final illustration of the results from the Keck aperture masking project, we show in Figure 3 diffraction limited maps of the enshrouded carbon star IRC +10216 taken in January 1997 at $2.26 \mu \mathrm{m}$ and $3.08 \mu \mathrm{m}$. First published in Danchi et al. (1997), these maps have the highest angular resolution to date, and furthermore show beautiful agreement with recent work of Weigelt et al. (1998) and Haniff \& Buscher (1998).

The maps show dramatic departure from the spherical symmetric outflow scenarios common in the literature, with pronounced plumes and clumps domi- 
nating the morphology. We believe that IRC +10216 has an underlying bipolar morphology with an axis of symmetry running at a position angle of about $20^{\circ}$ - an interpretation which is reinforced by the bipolar nature of the visible scattering nebula (Haniff \& Buscher 1998).
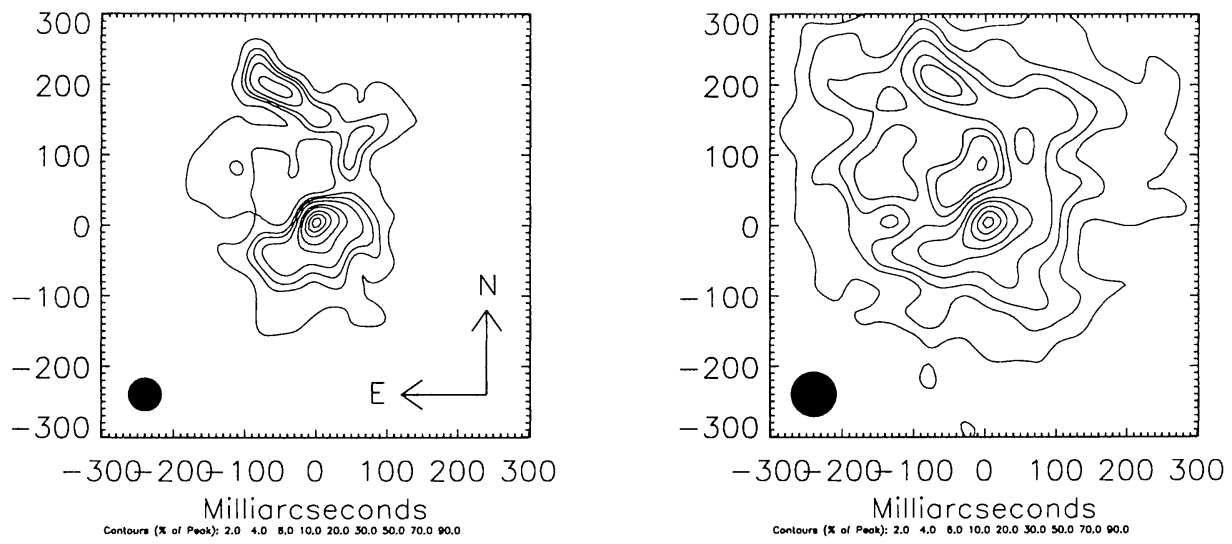

Figure 3. Contour maps of the enshrouded carbon star IRC +10216 from interferograms recorded in January 1997 at $2.26 \mu \mathrm{m}$ (left) and $3.08 \mu \mathrm{m}$ (right).

It is not certain which, if any, of the features visible in the maps corresponds to the central star. However if we make the tentative identification that the brightest (southern) component is the star itself, then we can derive an apparent diameter of about 50 mas for this object. This is in reasonable agreement with expectations based upon effective temperature and luminosity arguments.

Our study has also produced tentative evidence for time-evolution of the circumstellar environment of IRC +10216 by examining maps taken over a period from January 1997 through June 1998. The displacement between the bright southern feature (which we assume for the present to be the star itself) and the northern arm was seen to exhibit a motion of some 10 mas over a one year timescale. This is in agreement with expectations: if we take an outflow velocity of $14 \mathrm{~km} / \mathrm{sec}$ (Goldhaber 1988) and a distance to IRC +10216 of $290 \mathrm{pc}$ (Herbig \& Zappala 1970) we arrive at a linear motion of 10 mas per year. If, however, IRC +10216 is taken to lie at 150 pc (Crosas \& Menten 1997) then we must consider the possibility that the dust we see has a significant projected velocity on our line of sight (we only measure the tangential component in our proper motion study), or alternatively that the dust in the northern arm is still in a region where it is being accelerated by the radiation pressure and has not yet attained its terminal velocity. With continued monitoring, we should be able to answer these questions as to the detailed behavior of the outflow around this and a handful of other bright evolved stars. 


\section{References}

Born M., Wolf E., 1964, Principles of Optics, Macmillan, New York

Crosas M., Menten K.M., 1997, ApJ 483, 913

Danchi W.C., Tuthill P.G., Bester M., Lipman E.A., Monnier J.D., Townes C.H., 1997, in The Tenth Cambridge Workshop on Cool Stars, Stellar Systems, and the Sun, eds. R.A. Donahue \& J.A. Bookbinder, ASP Conf. Ser. 155,361

Goldhaber D.M., 1988, Ph.D. Dissertation, University of California, Berkeley Gull S.F., Skilling J., 1984, IEEE Proceedings 131, Pt. F, No. 6

Haniff C.A., Mackay C.D., Titterington D.J., Sivia D., Baldwin J.E., Warner P.J., 1987, Nature 328, 694

Haniff C.A., Buscher D.F., 1992, J. Opt. Soc. Am. A 9, 203

Haniff C.A., Scholz M., Tuthill P.G., 1995, MNRAS 266, 745

Haniff C.A., Buscher D.F., 1998, A\&A 334, L5

Herbig G.H., Zappala R.R., 1970, ApJ 162, L15

Högbom J., 1974, ApJS 15, 417

Matthews K., Soifer B.T., 1994, Ap\&SS 190, 239

Matthews K., Ghez A.M., Weinberger A.J., Neugebauer G., 1996, PASP 108, 615

Monnier J.D., Tuthill P.G., Lopez B., Cruzalèbes P., Danchi W.C., Haniff C.A., 1999, ApJ 512, 351

Roddier F., 1988, Physics Reports 170, 97

Sivia D.S., 1987, Ph.D. Dissertation, Cambridge University

Tuthill P.G., Haniff C.A., Baldwin J.E., 1997, MNRAS 285, 529

Weigelt G., Balega Y., Fleischer A.J., Osterbart R., Winters J.M., 1998, A\&A 333, L51 